is naturaleza 


\section{DOSSIER CRÍTICO SOBRE EL LIBRO:}

\section{LOS SÓTANOS DEL UNIVERSO}

Volumen monográfico de

\section{NATURALEZA Y LIBERTAD}

Revista de estudios interdisciplinares

Número 3

Málaga, 2014

Esta revista es accesible on-line en el siguiente portal: http://grupo.us.es/naturalezayl 


\section{Naturaleza y Libertad}

Revista de estudios interdisciplinares

Número 3, 2014

ISSN: 2254-96682014

Directores: Juan Arana, Universidad de Sevilla; Juan José Padial, Universidad de Málaga; Francisco Rodríguez Valls, Universidad de Sevilla.

Secretaria: Avelina Cecilia Lafuente, Universidad de Sevilla.

Consejo de Redacción: José Luis González Quirós, Universidad Juan Carlos I, Madrid; Francisco Soler, Universität Dortmund / Universidad de Sevilla; Pedro Jesús Teruel. Universidad CEU Cardenal Herrera; Héctor Velázquez, Universidad Panamericana, México.

Adjunto a la redacción: Miguel Palomo, Universidad de Sevilla

Consejo Editorial: Mariano Álvarez, Real Academia de Ciencia Morales y Políticas; Allan Franklin, University of Colorado; Michael Heller, Universidad Pontificia de Cracovia; Manfred Stöcker, Universität Bremen; William Stoeger, University of Arizona.

Consejo Asesor: Rafael Andrés Alemañ Berenguer. Universidad de Alicante; Juan Ramón Álvarez, Universidad de León; Luciano Espinosa, Universidad de Salamanca; Miguel Espinoza, Université de Strasbourg; Juan A. García González, Universidad de Málaga; José Manuel Giménez Amaya, Universidad de Navarra; Karim Gherab Martín, Urbana University, Illinois; Martín López Corredoira, Instituto de Astrofísica de Canarias; Alfredo Marcos, Universidad de Valladolid; Javier Monserrat, Universidad Autónoma de Madrid; Leopoldo Prieto, Colegio Mayor San Pablo, Madrid; Ana Rioja, Universidad Complutense, Madrid. Madrid; José Luis González Recio, Universidad Complutense, Madrid; Javier Serrano, TEC Monterrey (México); Hugo Viciana, Université Paris I; Claudia Vanney, Universidad Austral, Buenos Aires; José Domingo Vilaplana, Huelva.

\section{Redacción y Secretaría:}

Naturaleza y Libertad. Revista de estudios interdisciplinares. Departamento de Filosofía y Lógica. Calle Camilo José Cela s.n. E-41018 Sevilla.

Depósito Legal: MA2112-2012

용 954.55.77.57 Fax: 954.55.16.78. E-mail: jarana@us.es 
Naturaleza y Libertad. Revista de estudios interdisciplinares. Número 3, 2014. ISSN: 2254-9668

\section{ÍNDICE}

PRESENTACIÓN.

DOSSIER CRÍTICO SOBRE EL LIBRO LOS SÓTANOS DEL UNIVERSO

Juan Arana (U. de Sevilla), El problema de la determinación y el diálogo interdisciplinar......11

Miguel Acosta (U. CEU San Pablo, Madrid), La epistemología del riesgo y la

relación ciencia-filosofía. . .25

Carlos del Ama (Madrid), Reflexiones con ocasión del libro Los sótanos del universo ...........57

Luciano Espinosa (U. de Salamanca), De los sótanos al ático del universo ............................93

Gonzalo Génova (U. Carlos III, Madrid), Turing y el rostro del universo ..........................101

Julio A. Gonzalo (U. Autónoma de Madrid), De los sótanos a los confines del universo ....111

Juan Meléndez Sánchez (U. Carlos III, Madrid), De riesgos y metáforas

Andrés Muñoz Machado (Universidad Politécnica de Madrid), Las matemáticas en

Los sótanos del universo

Javier Sánchez Cañizares (U. de Navarra), Sótanos del universo, espejos del alma

Francisco José Soler Gil (U. de Sevilla), Una filosofía de la naturaleza contracorriente y antisistema.

Ignacio Sols (U. Complutense, Madrid), Recuperando la causa formal sin abandonar la causa eficiente. .151

Juan Arana (U. de Sevilla), Los subsótanos del universo. Materiales para un debate. 167 


\title{
DE RIESGOS Y METÁFORAS
}

\section{En torno a Los sótanos del universo}

\author{
Juan Meléndez Sánchez \\ Universidad Carlos III. Madrid
}

Resumen: Dos ecos galileanos percibidos en Los sótanos del universo: (1) La epistemología del riesgo que preconiza Juan Arana se prefigura en el cálculo de errores que inaugura (al menos conceptualmente) Galileo, y que se opone al paradigma del rigor escolástico que sigue arraigado en la filosofía de Descartes y sus sucesores. (2) La metáfora del conocimiento como resultado del "problema inverso" que resuelven los geólogos, infiriendo la estructura de la Tierra de los ecos de los terremotos, sugiere un paralelismo con el "subsuelo matemático" en el que residen las teorías para Galileo, y nos hace especular sobre la naturaleza matemática de la realidad última.

Palabras clave: Epistemología, Galileo, Escolástica, Descartes, Ciencia, Cálculo de errores, Matemáticas.

Abstract: Two Galilean echoes perceived in Los Sótanos del Universo: (1) The epistemology of risk precognised by Juan Arana is prefigured in the calculus of errors that Galileo inaugurate (conceptually at least) and that is opposed to the paradigm of scholastic rigour which is found in Descartes and in his successors' philosophy. (2) The metaphor of knowledge as result of the "inverse problem", which geologists solve deducing the estructure of the Earth from the echoes produced by earthquakes, suggests a similarity with the "underground of mathematics" where the theories are according to Galileo and that make us questioning about the mathematical nature of the final reality.

Keywords: Epistemology, Galileo, Scholasticism, Descartes, Science, Calculus of Errors, Mathematics.

Recibido: 18 de diciembre de 2012. Aprobado: 12 de diciembre de 2013. 
No había leído ningún libro del profesor Arana, aunque recuerdo haber hojeado en una librería hace tiempo un grueso volumen, que al final no me decidí a comprar a pesar de que su título me pareció admirable: Materia, Universo, Vida. Los sótanos del universo (LSU) es un título aún mejor, y un volumen aún mayor, y esta vez sí me he lanzado a leerlo.

Lamento no haberlo descubierto antes, porque me ha sorprendido una prosa suelta, un estilo desacomplejado y vivo incluso cuando es denso, como a menudo lo es. Pero lo mejor es que es un estilo que hace pensar, y que a mí me ha hecho ver cosas desde ángulos completamente nuevos. Por decir sólo un ejemplo: me ha parecido fantástica la conexión entre las mónadas de Leibniz y las funciones matemáticas en el $§ 30$.

Como tengo poco tiempo voy a limitarme a comentar dos ideas de las muchas que me ha sugerido este libro, y que me han llamado la atención seguramente por mi doble condición de físico y de aficionado a la historia de la ciencia.

La primera idea tiene que ver con la historia de la ciencia. Pese a que soy un mero aficionado, mi universidad, la Carlos III de Madrid, me dejó impartir allá por 2003 un curso de humanidades que titulé De Tales a Newton. Para mi sorpresa, ha tenido cierto éxito porque sigo impartiéndolo desde entonces, e incluso ha acabado materializándose en un libro (De Tales a Newton: Ciencia para personas inteligentes, Ellago Ediciones, 2013).

Pues bien, preparando el curso y el libro tuve ocasión de leer mucho sobre Galileo y aprendí algunas cosas curiosas. Por ejemplo, que él nunca dejó caer 
Naturaleza y Libertad. Revista de estudios interdisciplinares. Número 3, 2014. ISSN: 2254-9668

dos esferas de distinto material desde lo alto de la Torre de Pisa para demostrar que llegaban al suelo a la vez. Pero que a la vez la leyenda tiene algo de cierto, porque el famoso experimento sí se realizó en 1612, cuando Galileo ya estaba en Florencia, por un profesor aristotélico llamado Giorgio Coresio. Y lo más curioso del caso es que Coresio llegó a la conclusión de que el resultado desmentía a Galileo, porque en realidad los cuerpos más pesados llegaban al suelo un poco antes.

Leyendo LSU he recordado este episodio y he pensado que el análisis del aristotélico Coresio es un ejemplo de las trampas del paradigma del rigor del que habla el profesor Arana, ese rigor mal entendido que a fuerza de aceptar sólo verdades incontrovertibles ha venido carcomiendo a la filosofía desde Descartes. Cuando Coresio niega que los dos pesos caigan a la vez porque no lo hacen exactamente a la vez, me parece que hace en el fondo lo mismo que Descartes con su duda metódica, que en esencia es "tener por falsas las cosas dudosas" (como leemos en la p. 31 de LSU [§ 8]). Es decir, niega que algo sea verdad porque no es palmariamente evidente. Quizá Descartes, que aborrecía a sus maestros escolásticos, no estaba en sus actitudes tan lejos de ellos. En este libro se explica muy bien que despertar del sueño dogmático no es tan sencillo.

Contra el rigor (rigor mortis) de los escolásticos, Galileo reacciona con el vigoroso sentido común de un comerciante, y por boca de Salviati da una respuesta memorable:

¿Sabes lo que sucede, Simplicio? De la misma forma que el calculador que quiere que sus cálculos sean sobre el azúcar, seda y lana debe descontar las cajas, embalajes y otras envolturas, así el científico, cuando quiere reconocer en concreto los efectos que ha demostrado 
Dossier crítico sobre el libro: Los sótanos del universo

en abstracto, debe restar los obstáculos materiales; y si es capaz de hacer esto, te aseguro que las cosas no tienen menos acuerdo que los cómputos aritméticos.

Hasta que no se reconoció la importancia de este factor y no se supo tratar no fue posible la ciencia experimental. Saber descontar los embalajes es todo un arte que en física llamamos cálculo de errores; un arte que intentamos enseñar a los alumnos en los laboratorios. Casi siempre con poco éxito, la verdad, porque todos los años nos encontramos resultados con ocho cifras de precisión: el espejismo del rigor no es sólo un vicio de filósofos sino que tiene raíces muy profundas

Por eso tiene un mérito extraordinario la perspicacia de Galileo, que fue quien primero entendió que es posible hacer una ciencia no exacta, y no sólo que es posible sino que es la única manera de hacer una ciencia que no tenga, como dice el profesor Arana (en la p. 43 [§ 13]) "el germen de una muerte precoz" en su dotación genética. De modo que resumiendo mi primera idea, quiero proponer a Galileo como el patrón de la epistemología del riesgo que tan necesariamente reivindica Juan Arana en este libro.

\section{2}

La segunda idea es más bien una especulación hilvanando una serie de metáforas que empiezan por el propio título del libro, Los sótanos del universo. Me ha parecido una imagen brillante para expresar que la maquinaria de la causación, o para ser precisos, como aquí se dice, de la determinación, permanece oculta, en un subsuelo inaccesible a nosotros. Aunque vemos lo que se hace y quién o qué lo hace, nunca conseguimos atisbar cómo se hace, como ocurre con 
los buenos ilusionistas (p. 24). Pero el "nunca" es relativo, porque, como leemos a continuación, (§ 5)

Los geólogos no tienen la posibilidad de perforar un pozo hasta el centro de la Tierra para comprobar su textura y consistencia. Se tienen que conformar con estudiar los ecos de terremotos ocurridos en las antípodas para conjeturar estructuras que escapan a nuestro control directo. Algo parecido deberá hacer quien se pregunte por la verdad escondida detrás de todo lo que da unidad al universo.

Creo que la imagen de los geólogos enriquece mucho la metáfora original, porque, efectivamente, toda la investigación científica es como la de los geólogos, aunque pueda no parecerlo. El experimento es una pregunta que hacemos a la naturaleza. Pero ¿cómo podemos preguntarle? La naturaleza no habla castellano: la única manera de hacerle una pregunta es perturbándola, igual que las ondas del terremoto de las antípodas perturban el núcleo terrestre. Y la reacción a esta perturbación es la respuesta. Del conjunto de las respuestas de la naturaleza a nuestras preguntas, de los resultados de nuestros experimentos, intentamos inferir su estructura. Es decir, todos hacemos ingeniería inversa como los geólogos. Pero esto sólo es posible si tenemos un modelo tentativo de esa estructura.

Esto nos lleva de nuevo a Galileo, que fue el primero que tuvo éxito en hacer ciencia porque se dio cuenta de esto y trabajó constantemente con modelos matemáticos. La metáfora del subsuelo me ha recordado a otra de mi cosecha que uso en mi curso para explicar el método científico tal como Galileo lo entendía. Es un proceso que parte de los fenómenos, pero que no se entretiene en coleccionarlos, como postulaban los empiristas, sino que tan pronto como puede hace un modelo matemático de ellos. Ese modelo ya no 
está en el mundo sensible: pertenece al mundo de las matemáticas. Yo pido a los alumnos que se lo imaginen como el subsuelo de una ciudad, y a las herramientas y resultados de las matemáticas como infraestructuras, túneles del metro que nos permiten viajar con mucho menos esfuerzo que por la superficie. Así, nuestro modelo puede llegar muy lejos; es decir, podemos extraer de él consecuencias nada obvias; y algunas de ellas las podremos concretar como predicciones para un experimento, que realizaremos al final del viaje, ya fuera del subsuelo.

Este proceso de modelización matemática, derivación de predicciones y contrastación con la realidad, se repite una y otra vez, poniendo así constantemente a prueba el modelo.

Pero creo que Galileo iba más lejos. Para él, que el modelo sea matemático no es una cuestión de mera conveniencia, "para que viaje más lejos en el metro". El modelo tiene que ser matemático porque la realidad lo es: es el lenguaje en el que está escrito el libro de la naturaleza.

En LSU esto se menciona en la p. 107 [§ 42] y me llama la atención que enseguida aparecen dos figuras, dos estrellas, en las que las leyes físicas son como líneas que conectan las formas accidentales en la periferia de la realidad, pasando por el núcleo de la forma sustancial, la esencia. Al ver este dibujo no pude evitar acordarme de los geólogos, imaginar la Tierra como esa estrella cuyo núcleo queremos conocer y ver esas líneas como las ondas de los terremotos. Más aún cuando se dice en la p. 109 [§ 43] que "la red de relaciones que tejen las leyes fisicomatemáticas horada la cáscara superficial de los fenómenos y sondea a mayor o menor profundidad el invisible en sí de los objetos". 
Naturaleza y Libertad. Revista de estudios interdisciplinares. Número 3, 2014. ISSN: 2254-9668

Esta frase me ha impresionado y voy a terminar con una especulación que me ha sugerido, aún a riesgo de que puedan pensar que, como al hidalgo los libros de caballería, tanta metáfora me ha secado el cerebro.

Una red de relaciones no es más que forma. Y formas y relaciones son la sustancia de la matemática. ¿No podría ser que el en sí de los objetos fueran precisamente esas relaciones, que no hubiera "cosas" sino sólo estructura y que la realidad fuese matemática como creía Galileo, pero en un sentido más fuerte que el que solemos dar a su frase sobre el Libro; en un sentido, en definitiva, netamente pitagórico?

Muchas gracias por su atención. Prometo estar a dieta de metáforas una buena temporada.

Juan Meléndez Sánchez melendez@fis.uc3m.es 
